\title{
High-precision determination of the critical exponent $\gamma$ for self-avoiding walks
}

\author{
Sergio Caracciolo \\ Scuola Normale Superiore and INFN - Sezione di Pisa \\ I-56100 Pisa, ITALIA \\ Internet: Sergio.Caracciolo@sns.it \\ Maria Serena Causo \\ Dipartimento di Fisica and INFN - Sezione di Lecce \\ Università degli Studi di Lecce \\ I-73100 Lecce, ITALIA \\ Internet: causo@le.infn.it \\ Andrea Pelissetto \\ Dipartimento di Fisica and INFN - Sezione di Pisa \\ Università degli Studi di Pisa \\ I-56100 Pisa, ITALIA \\ Internet: pelisset@ibmth.difi.unipi.it
}

\begin{abstract}
We compute the exponent $\gamma$ for self-avoiding walks in three dimensions. We get $\gamma=1.1575 \pm 0.0006$ in agreement with renormalization-group predictions. Earlier Monte Carlo and exact-enumeration determinations are now seen to be biased by corrections to scaling.
\end{abstract}


The self-avoiding walk (SAW) is a well-known lattice model of a polymer molecule in a good solvent; moreover, because of its semplicity, it is an important test-case in the theory of critical phenomena. Much work has been done in computing critical exponents by a variety of theoretical approaches - Monte Carlo (MC), exact enumeration/extrapolation (EE), and renormalization group (RG) - with the aim of comparing these determinations with each other and with the experimental results. Small but persistent discrepancies have emerged between the predictions from different theoretical approaches. For example, extensive studies have been done on the exponent $\nu$ which controls the critical behaviour of the length scales. Early MC simulations and EE studies [1] in the ' 70 s predicted $\nu=3 / 5$ in agreement with the Flory theory. When the length of the walks which were simulated was increased [2, 3, [4] the value of $\nu$ decreased to $\nu=0.592 \pm 0.002$. This value was also supported by extended EE studies which provided an identical estimate [5]. On the other hand, field-theoretic RG computations [6, 7, 8, 9, 10] persistently gave $\nu=0.588 \pm 0.001$ or even slightly lower. The discrepancy was clarified when the MC studies were extended to much longer walks: because of strong corrections with non-analytic exponent $\Delta \approx 0.5$, the asymptotic regime is reached only for very long chains, and the results from shorter chains are systematically biased upward [11, 12, 13, 14]. A simulation [14] with walks of length up to $N=80000$, using a data analysis taking careful account of the corrections to scaling, gave $\nu=0.5877 \pm 0.0006$ in good agreement with the renormalization-group estimates. Also universality is well satisfied: a simulation 13] in a slightly different geometry provided $\nu=0.5867 \pm 0.0013$ (68\% confidence limit) while a recent high-statistics simulation [15] for the off-lattice Kratky-Porod model with excluded volume gave $\nu=0.5880 \pm 0.0018$. One may ask if the same phenomenon occurs for the other critical exponents. For the exponent $\gamma$, there are indeed significant discrepancies in the existing theoretical predictions. $\mathrm{MC}$ and $\mathrm{EE}$ studies provide the estimates

$$
\gamma= \begin{cases}1.161 \pm 0.001 & \text { EE, Ref. [5] } \\ 1.1619 \pm 0.0001 & \text { EE, Ref. 17 } \\ 1.1608 \pm 0.0003 & \text { MC, Ref. 16 }\end{cases}
$$

On the other hand the $\epsilon$-expansion predicts a lower value [7]: $1.157 \lesssim \gamma \lesssim 1.160$. Indeed a Borel-type resummation gives $\gamma=1.160 \pm 0.004$ and $\gamma=1.157 \pm 0.003$ if one forces the expansion to reproduce the exact value in two dimensions $\gamma=\frac{43}{32}$. One can also use the scaling relation $\gamma=(2-\eta) \nu$ and the estimates for $\eta$ and $\nu$ : the unconstrained $\epsilon$-expansion gives $\nu=0.5885 \pm 0.0025$ and $\eta=0.031 \pm 0.003$ so that $\gamma=1.159 \pm 0.005$; using the exactly known values for $d=2, \nu=\frac{3}{4}$ and $\eta=\frac{5}{24}$, one gets $\nu=0.5880 \pm 0.0015$ and $\eta=0.0320 \pm 0.0025$ so that $\gamma=1.1572 \pm 0.0035$. More controversial is the status of the expansions at fixed spatial dimension $d=3$. In [7] $\gamma$ is estimated as $\gamma=1.1615 \pm 0.0020$ while in [9] the final estimate is $\gamma \approx 1.1613$. These estimates depend crucially on the critical value of the renormalized coupling constant $\bar{g}^{*}$ : in [6, 7] the estimate $\bar{g}^{*}=1.421 \pm 0.008$ is used, while [9] uses $\bar{g}^{*}=1.422 \pm 0.008$. However Nickel [18] has pointed out that the present estimates of $\bar{g}^{*}$ could be slightly higher than the correct value due to a possible non-analyticity of the $\beta$-function at $\bar{g}^{*}$ which is usually neglected in the standard analyses. A reanalysis of the series 
110 indicates that $\bar{g}^{*}$ could be as low as 1.39 and predicts $\gamma=1.1569+0.10\left(\bar{g}^{*}-\right.$ $1.39) \pm 0.0004$. In this paper we present a high-precision MC study for the exponent $\gamma$ using walks up to length $N=40000$. Our results confirm the important role played by corrections to scaling; our final estimate $\gamma=1.1575 \pm 0.0006$ is significantly lower than previous $\mathrm{MC}$ and EE results but it is in perfect agreement with the RG predictions. Moreover it clearly supports the analysis by Nickel [18, 10] and the value $\bar{g}^{*} \approx 1.39$ for the renormalized coupling constant. In presence of strong corrections to scaling, in order to get a reliable estimate of the critical exponents, one needs to perform the simulation in the large- $N$ regime. This is only possible if the algorithm at hand does not exhibit too strong a critical slowing-down. For the study of $\gamma$ for SAWs on the lattice the best available algorithm is the join-and-cut algorithm [19]: in two dimensions the autocorrelation time, expressed in CPU units, behaves approximately as $N^{\approx 1.5}$ while in three dimensions the behaviour is expected to be $N^{\approx 1.2}$. The algorithm is thus nearly optimal. Another advantage of this algorithm is that it does not require the determination of the connectivity constant, at variance with more standard algorithms. The join-and-cut algorithm works in the unorthodox ensemble $T_{N_{t o t}}$ consisting of all pairs of SAWs (each walk starts at the origin and ends anywhere) such that the total number of steps in the two walks is some fixed number $N_{\text {tot }}$. Each pair in the ensemble is given equal weight: therefore the two walks are not interacting except for the constraint on the sum of their lengths. One sweep of the algorithm consists of two steps:

1. Starting from a pair of walks $\left(\omega_{1}, \omega_{2}\right)$, we update each of them independently using some ergodic fixed-length algorithm. We use the pivot algorithm [20, 21, 3] which is the best available one for the ensemble of fixed-length walks with free endpoints.

2. We perform a join-and-cut move: we concatenate the two walks $\omega_{1}$ and $\omega_{2}$ forming a new (not necessarily self-avoiding) walk $\omega_{\text {conc }}$; then we cut $\omega_{\text {conc }}$ at a random position creating two new walks $\omega_{1}^{\prime}$ and $\omega_{2}^{\prime}$. If $\omega_{1}^{\prime}$ and $\omega_{2}^{\prime}$ are selfavoiding we keep them; otherwise the move is rejected and we stay with $\omega_{1}$ and $\omega_{2}$.

More details on the dynamical critical behaviour and on the implementation of this algorithm can be found in [19]. Let us now discuss how the critical exponent $\gamma$ can be estimated from the Monte Carlo data produced by the join-and-cut algorithm. Let us start by noticing that the random variable $N_{1}$, the length of the first walk, has the distribution

$$
\bar{\pi}\left(N_{1}\right)=\frac{c_{N_{1}} c_{N_{t o t}-N_{1}}}{Z\left(N_{t o t}\right)}
$$

for $1 \leq N_{1} \leq N_{\text {tot }}-1$ where $Z\left(N_{\text {tot }}\right)$ is the obvious normalization factor and $c_{N}$ is the number of walks going from the origin to any lattice point with $N$ steps. For large $N$ we have

$$
c_{N} \approx a_{0} \mu^{N} N^{\gamma-1}
$$

and thus the idea is to make inferences of $\gamma$ from the observed statistics of $N_{1}$. Of course the problem is that (3) is an asymptotic formula valid only in the large- $N$ 
regime. We will thus proceed in the following way: we will suppose that (3) is valid for all $N \geq N_{\min }$ for many increasing values of $N_{\min }$ and correspondingly we will get estimates $\hat{\gamma}\left(N_{\text {min }}\right)$; these quantities are effective exponents which depend on $N_{\text {min }}$ and which give correct estimates of $\gamma$ as $N_{\text {min }}$ and $N_{\text {tot }}$ go to infinity. The determination of $\gamma$ from the data is obtained using the maximum-likelihood method. We will present here only the results: for a detailed discussion we refer the reader to 19. Given $N_{\text {min }}$ consider the function

$$
\theta_{N_{\min }}\left(N_{1}\right)= \begin{cases}1 & \text { if } N_{\min } \leq N_{1} \leq N_{t o t} \\ 0 & \text { otherwise }\end{cases}
$$

and let $X$ be the random variable

$$
X=\log \left[N_{1}\left(N_{t o t}-N_{1}\right)\right]
$$

Then define

$$
X^{\text {cens }}\left(N_{\text {min }}\right)=\frac{\left\langle X \theta_{N_{\text {min }}}\right\rangle}{\left\langle\theta_{N_{\text {min }}}\right\rangle}
$$

where the average $\langle\cdot\rangle$ is taken in the ensemble $T_{N_{\text {tot }}}$ sampled by the join-and-cut algorithm. The quantity defined in (6) is estimated in the usual way from the Monte Carlo data obtaining is this way $X_{M C}^{c e n s}\left(N_{\text {min }}\right)$. Then $\hat{\gamma}\left(N_{\text {min }}\right)$ is computed by solving the equation

$$
X_{M C}^{c e n s}\left(N_{\min }\right)=[X]_{t h, \hat{\gamma}}\left(N_{\min }\right)
$$

where, for every function of $N_{1}$, we define

$$
\left[f\left(N_{1}\right)\right]_{t h, \gamma}\left(N_{\min }\right) \equiv \frac{\sum_{N_{1}=N_{\min }}^{N_{t o t}-N_{\min }} f\left(N_{1}\right) N_{1}^{\gamma-1}\left(N_{t o t}-N_{1}\right)^{\gamma-1}}{\sum_{N_{1}=N_{\min }}^{N_{t o t}-N_{\min }} N_{1}^{\gamma-1}\left(N_{t o t}-N_{1}\right)^{\gamma-1}}
$$

The variance of $\hat{\gamma}\left(N_{\text {min }}\right)$ is then given by

$$
\operatorname{Var}\left[\hat{\gamma}\left(N_{m i n}\right)\right]=\frac{\operatorname{Var}\left(X_{M C}^{c e n s}\left(N_{m i n}\right)\right)}{\left([X ; X]_{t h, \hat{\gamma}}\left(N_{m i n}\right)\right)^{2}}
$$

where $[X ; X]=\left[X^{2}\right]-[X]^{2}$. We must finally compute $\operatorname{Var}\left(X_{M C}^{\text {cens }}\left(N_{\text {min }}\right)\right)$. As this quantity is defined as the ratio of two mean values (see formula (6)) one must take into account the correlation between denominator and numerator. Here we have used the standard formula for the variance of a ratio (valid in the large-sample limit)

$$
\operatorname{Var}\left(\frac{A}{B}\right)=\frac{\langle A\rangle^{2}}{\langle B\rangle^{2}} \operatorname{Var}\left(\frac{A}{\langle A\rangle}-\frac{B}{\langle B\rangle}\right)
$$

Finally let us mention how to combine data from runs at different values of $N_{\text {tot }}$. The approach we use consists in analyzing the data separately for each $N_{\text {tot }}$ and then in constructing the usual weighted average of the resulting estimates $\hat{\gamma}\left(N_{\text {min }}\right)$ with weights inversely proportional to the estimated variances. Let us now discuss our results. We have performed high-statistics runs at $N_{t o t}=200,2000,20000$ and 40000 . The number of iterations is reported in Table 1. The total simulation took 16 months 
on an AlphaStation 600 Mod 5/266. In the same table we report also, for two different values of $N_{\text {min }}$, the autocorrelation times for the observable

$$
Y\left(N_{\text {min }}\right)=\frac{X \theta_{N_{\text {min }}}}{\left\langle X \theta_{N_{\text {min }}}\right\rangle}-\frac{\theta_{N_{\text {min }}}}{\left\langle\theta_{N_{\text {min }}}\right\rangle}
$$

which according to (10) controls the errors on $\gamma$. We use here a standard autocorrelation analysis [3] with a self-consistent window of $15 \tau_{\text {int }, Y}$, supplemented by an ad hoc prescription to take into account tails in the autocorrelation functions [14]. The contribution of the tail to $\tau_{\text {int }, Y}$ amounts to approximately $20 \%$ for the two larger values of $N_{t o t}, 2 \%$ for $N_{t o t}=2000$, while for $N_{t o t}=200$ we have been unable to identify any tail. The results are consistent with the expectation of $\tau_{\text {int }, Y} \sim N \approx 0.3$ so that, keeping into account that the CPU-time per iteration increases as $\sim N^{\approx 0.9}$, we find that the CPUtime per independent walk increases roughly as $N^{\approx 1.2}$. Our raw data are reported in Table 2 and Table 3 where we give for various $N_{\min }$ the estimates of $X_{M C}^{c e n s}\left(N_{\min }\right)$. Let us now look at the results. Let us consider first $N_{t o t}=200$. We see here that the estimates of $\gamma$ increase with $N_{\min }$ and for $N_{\min }=50$ they give $\gamma \approx 1.1605$. This value is in agreement with Monte Carlo studies [16] performed in the same range of values of $N$ and exact-enumeration studies [5, 17]. Consider now $N_{\text {tot }}=2000$. One can see that the estimates of $\gamma$ are lower and indicate $1.1580 \lesssim \gamma \lesssim 1.1585$ : clearly the estimate at $N_{\text {tot }}=200$ was biased upward by the corrections to scaling. Let us now consider the results of Table 3 where we give the estimates of $\gamma$ coming from the weighted average of the results with $N_{t o t}=20000$ and $N_{t o t}=40000$. The estimates are extremely flat and agree within error bars from $N_{\min }=1$ to $N_{\min }=8000$; they indicate $1.1574 \lesssim \gamma \lesssim 1.1578$. Again the new estimate is lower than the previous ones. At least at $N_{t o t}=2000$ there are still systematic deviations which are larger than the statistical error. Of course the same could be true for our results at the larger value of $N_{\text {tot }}$. The most straightforward way to solve the question would be to have data at a higher value of $N_{\text {tot }}$, say $N_{\text {tot }}=4 \cdot 10^{5}$, with comparable statistics. However this is not possible with current computer resources. We have thus simply tried to estimate the systematic bias by comparing the results with different $N_{\text {tot }}$ assuming that the systematic error vanishes as $N_{\text {tot }}^{-1 / 2}$ (this is the behaviour one expects if the corrections to $c_{N}$, formula (3), vanish as $N^{-1 / 2}$ ). In this way we get an estimate of 0.0002-0.0003 as the residual bias on the data at $N_{\text {tot }}=40000$. We have thus taken as our final estimate the value at $N_{\min }=2000$ :

$$
\gamma=1.1575 \pm 0.0003 \pm 0.0003
$$

where the first error is the statistical one (68\% confidence limits) while the second is a subjective estimate of the error due to the corrections to scaling. Let us finally discuss the determinations of the connectivity constant $\mu$ which is usually derived together with the estimate of $\gamma$. Restricting ourselves to the case of the cubic lattice - the discussion is completely analogous in the other cases - EE and MC studies give the following estimates for $\beta_{c} \equiv 1 / \mu$ :

$$
\beta_{c}= \begin{cases}0.213496 \pm 0.000004 & \text { EE, Ref. [5] } \\ 0.213499 \pm 0.000001 & \text { EE, Ref. [17] } \\ 0.213492 \pm 0.000001 & \text { MC, Ref. [16 }\end{cases}
$$




\begin{tabular}{|c|c|c|c|}
\hline$N_{\text {tot }}$ & $N_{\text {iter }}$ & $\tau_{\text {int }, Y(1)}$ & $\tau_{\text {int }, Y(1000)}$ \\
\hline \hline 200 & $5 \cdot 10^{8}$ & $1.47970 \pm 0.00055$ & - \\
2000 & $6.2 \cdot 10^{8}$ & $2.808 \pm 0.022$ & - \\
20000 & $10^{8}$ & $6.96 \pm 0.18$ & $9.35 \pm 0.21$ \\
40000 & $8.5 \cdot 10^{8}$ & $8.80 \pm 0.23$ & $10.78 \pm 0.24$ \\
\hline
\end{tabular}

Table 1: Number of iterations and autocorrelation times for the various values of $N_{t o t}$.

However these results have probably a large systematic error as the corresponding estimates of $\gamma$ are quite far from the correct value. To give an estimate of this effect we have considered the series

$$
S(\delta)=\left[\sum_{N=0}^{\infty} c_{N} \beta^{N}\right]^{\frac{1}{\delta}}
$$

where $c_{N}$ is the number of walks of length $N$. If $\delta=\gamma, S(\delta)$ has a simple pole as $\beta=\beta_{c}$ and thus, neglecting subleading corrections, $\beta_{c}$ can be determined by a simple Padè analysis. Using the values of $c_{N}$ reported in [5, 17], we find

$$
\beta_{c}= \begin{cases}0.2134987 \pm 0.0000001 & \text { for } \delta=1.1619 \\ 0.2134811 \pm 0.0000031 & \text { for } \delta=1.1576\end{cases}
$$

where the "error" indicates the spread of the Padè approximants we have considered. Thus if use the value of $\gamma$ given by [17], see (1), we get a value for $\beta_{c}$ in agreement with their estimate (13). However if we use our estimate of $\gamma$, we get a $\beta_{c}$ which is off by five to twenty times the stated error bars. Of course we cannot trust (15) as a good estimate of $\beta_{c}$ as the analysis still does not take into account corrections to scaling; however this simple exercise shows that $\beta_{c}$ is lower than expected. It should also be noticed that this trend is already visible in (13) where $\beta_{c}$ obtained by MC is lower that the EE estimates. We thank Alan Sokal and Ettore Vicari for useful

discussions.

\section{References}

[1] M. G. Watts, J. Phys. A7, 489 (1974).

[2] D. C. Rapaport, J. Phys. A18, 113 (1985).

[3] N. Madras and A. D. Sokal, J. Stat. Phys. 50, 109 (1988).

[4] N. Eizenberg and J. Klafter, J. Chem. Phys. 99, 3976 (1993).

[5] A. J. Guttmann, J. Phys. A22, 2807 (1989). 


\begin{tabular}{|c|c|c||c|c|c|}
\hline & \multicolumn{2}{|c||}{$N_{\text {tot }}=200$} & & \multicolumn{2}{c|}{$N_{\text {tot }}=2000$} \\
\hline$N_{\min }$ & \multicolumn{1}{|c|}{$X_{M C}^{\text {cens }}$} & \multicolumn{1}{|c|}{$\gamma$} & $N_{\min }$ & \multicolumn{1}{c|}{$X_{M C}^{\text {cens }}$} & $\gamma$ \\
\hline 1 & $8.701168 \pm 0.000052$ & $1.15288 \pm 0.00011$ & 1 & $13.298266 \pm 0.000068$ & $1.15782 \pm 0.00013$ \\
10 & $8.842084 \pm 0.000036$ & $1.15808 \pm 0.00021$ & 100 & $13.452571 \pm 0.000046$ & $1.15802 \pm 0.00028$ \\
20 & $8.943002 \pm 0.000026$ & $1.15866 \pm 0.00034$ & 200 & $13.551931 \pm 0.000033$ & $1.15811 \pm 0.00045$ \\
30 & $9.017434 \pm 0.000019$ & $1.15875 \pm 0.00053$ & 300 & $13.625478 \pm 0.000025$ & $1.15838 \pm 0.00071$ \\
40 & $9.074675 \pm 0.000014$ & $1.15999 \pm 0.00084$ & 400 & $13.682081 \pm 0.000018$ & $1.1598 \pm 0.0011$ \\
50 & $9.119083 \pm 0.000010$ & $1.1605 \pm 0.0014$ & 500 & $13.725970 \pm 0.000013$ & $1.1584 \pm 0.0019$ \\
\hline
\end{tabular}

Table 2: Raw data and estimates of $\gamma$ for $N_{t o t}=200$ and $N_{t o t}=2000$.

\begin{tabular}{|c||c|c||c|}
\hline$N_{\min }$ & $X_{M C}^{\text {cens }}(20000)$ & $X_{M C}^{\text {cens }}(40000)$ & $\gamma$ \\
\hline 1 & $17.90254 \pm 0.00026$ & $19.28850 \pm 0.00010$ & $1.15763 \pm 0.00018$ \\
200 & $17.94330 \pm 0.00023$ & $19.310211 \pm 0.000095$ & $1.15762 \pm 0.00021$ \\
400 & $17.97715 \pm 0.00021$ & $19.329406 \pm 0.000090$ & $1.15758 \pm 0.00024$ \\
600 & $18.00687 \pm 0.00020$ & $19.346998 \pm 0.000086$ & $1.15765 \pm 0.00026$ \\
800 & $18.03376 \pm 0.00019$ & $19.363292 \pm 0.000083$ & $1.15760 \pm 0.00028$ \\
1000 & $18.05830 \pm 0.00017$ & $19.378624 \pm 0.000080$ & $1.15764 \pm 0.00030$ \\
1200 & $18.08095 \pm 0.00016$ & $19.393077 \pm 0.000077$ & $1.15754 \pm 0.00032$ \\
1400 & $18.10198 \pm 0.00015$ & $19.406824 \pm 0.000074$ & $1.15749 \pm 0.00034$ \\
1600 & $18.12172 \pm 0.00014$ & $19.419918 \pm 0.000071$ & $1.15744 \pm 0.00036$ \\
1800 & $18.14022 \pm 0.00013$ & $19.432477 \pm 0.000069$ & $1.15755 \pm 0.00038$ \\
2000 & $18.15760 \pm 0.00012$ & $19.444488 \pm 0.000067$ & $1.15749 \pm 0.00040$ \\
2200 & $18.17394 \pm 0.00012$ & $19.456041 \pm 0.000065$ & $1.15746 \pm 0.00042$ \\
2400 & $18.18930 \pm 0.00011$ & $19.467169 \pm 0.000063$ & $1.15739 \pm 0.00045$ \\
2600 & $18.20388 \pm 0.00011$ & $19.477901 \pm 0.000061$ & $1.15741 \pm 0.00047$ \\
2800 & $18.21771 \pm 0.00010$ & $19.488255 \pm 0.000059$ & $1.15739 \pm 0.00050$ \\
3000 & $18.230811 \pm 0.000093$ & $19.498275 \pm 0.000058$ & $1.15748 \pm 0.00052$ \\
3500 & $18.260909 \pm 0.000081$ & $19.521898 \pm 0.000053$ & $1.15753 \pm 0.00058$ \\
4000 & $18.287471 \pm 0.000069$ & $19.543763 \pm 0.000049$ & $1.15772 \pm 0.00066$ \\
5000 & $18.331323 \pm 0.000049$ & $19.582986 \pm 0.000043$ & $1.15749 \pm 0.00083$ \\
6000 & $18.364968 \pm 0.000034$ & $19.617183 \pm 0.000037$ & $1.1566 \pm 0.0010$ \\
7000 & $18.389939 \pm 0.000021$ & $19.647298 \pm 0.000032$ & $1.1586 \pm 0.0013$ \\
8000 & $18.407212 \pm 0.000011$ & $19.673754 \pm 0.000027$ & $1.1582 \pm 0.0017$ \\
\hline
\end{tabular}

Table 3: Raw data for $N_{t o t}=20000$ and $N_{t o t}=40000$ and combined estimate of $\gamma$ for various values of $N_{\text {min }}$. 
[6] J. C. Le Guillou and J. Zinn-Justin, Phys. Rev. B21, 3976 (1980).

[7] J. C. Le Guillou and J. Zinn-Justin, J. Physique Lett. 46, L137 (1985); J. Physique 50, 1365 (1989).

[8] J. des Cloizeaux, R. Conte and G. Jannink, J. Physique Lett. 46, L595 (1985).

[9] M. Muthukumar and B. G. Nickel, J. Chem. Phys. 86, 460 (1987).

[10] D. B. Murray and B. G. Nickel, 1991, unpublished.

[11] I. Majid, Z. V. Djordjevic and H. E. Stanley, Phys. Rev. Lett. 51, 1282 (1983).

[12] G. Zifferer, Makromoleculare Chem. 191, 2717 (1990); 192, 1555 (1991).

[13] S. Caracciolo, G. Ferraro and A. Pelissetto, J. Phys. A24, 3625 (1991).

[14] B. Li, N. Madras and A. D. Sokal, J. Stat. Phys. 80, 661 (1995).

[15] J. S. Pedersen, M. Laso and P. Shurtenberger, Phys. Rev. E54, R5917 (1996).

[16] P. Grassberger, J. Phys. A26, 2769 (1993).

[17] D. MacDonald, D. L. Hunter, K. Kelly and N. Jan, J. Phys. A25, 1429 (1992).

[18] B. G. Nickel, Physica A177, 189 (1991).

[19] S. Caracciolo, A. Pelissetto and A. D. Sokal, J. Stat. Phys. 67, 65 (1992).

[20] M. Lal, Molec. Phys. 17, 57 (1969).

[21] B. MacDonald, N. Jan, D. L. Hunter and M. O. Steinitz, J. Phys. A18, 2627 (1985). 